\title{
HOMEOPATIA E DESEMPENHO DE NOVILHOS CONFINADOS COM DIETA $100 \%$ OU 48 \% DE CONCENTRADO
}

\author{
EFFECT OF HOMEOPATHY ON PERFORMANCE OF FEEDLOT STEERS FEED WITH \\ DIETS CONTAINING $100 \%$ OR $48 \%$ CONCENTRATE
}

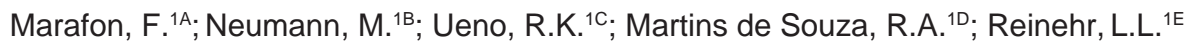 \\ e Poczynek, M. ${ }^{1 F}$
}

\begin{abstract}
${ }^{1}$ Universidade Estadual do Centro Oeste. Guarapuava, PR. Brasil. Afabiano_marafon@hotmail.com; Bmikaelneumann@hotmail.com; Crobsonueno@hotmailcom; Drdesouza@unicentro.com.br; Ereinehrllr@hotmail.com; Fmpoczynek@hotmail.com
\end{abstract}

PaLAVRAS ChaVe ADICIONAIS

Conversão alimentar. Ganho de peso. Hipófise.

\section{RESUMO}

O experimento teve como objetivo avaliar o consumo diário de matéria seca e o desempenho de novilhos terminados em confinamento utilizando dietas com 38 ou $100 \%$ de concentrado e homeopatia. Utilizou-se 32 novilhos Charolês inteiros, provenientes do mesmo rebanho, com idade média de 12 meses e peso vivo médio inicial de $323 \pm 5 \mathrm{~kg}$. Animais que consumiram dietas com $48 \%$ de concentrado apresentaram maiores ganhos de peso e melhor conversão alimentar com a inclusão da homeopatia. A homeopatia não manifestou resposta positiva com dieta $100 \%$ concentrado.

\section{SUMMARY}

The experiment aimed to evaluate the daily intake of dry matter and performance of feedlot finished young bulls with different inclusions of concentrate and homeopathy on the diet. Thirty two young Charolais bulls, coming from the same herd, with mean age of 12 months and average weight of $323 \pm 5 \mathrm{~kg}$ were used. Animals eating levels of $48 \%$ of concentrated showed better weight gain and feed conversion with homeopathy. The homeopathy has not showed positive response in animals finished in feedlot with $100 \%$ concentrate diet.

\section{INTRODUÇÃO}

O crescimento dos confinamentos no

\section{AdDitiOnAL KEYWORDS}

Feed conversion. Weight gain. Pituitary gland.

Brasil, juntamente com o aumento da produção agrícola, somam fatores que vêm contribuindo com a maior utilização de grãos e seus coprodutos na elaboração das dietas, nesse sentido, dietas isentas da fração volumosa passaram ser uma constante nos confinamentos brasileiros pela acessibilidade de preço das commodities (Ueno, 2012). Pouco se sabe ainda sobre a eficiência biológica que pode ser obtida com esta tecnologia. A homeopatia visa diminuir a permanência dos animais em regime de estabulamento, com vistas também a não agressão do meio ambiente.

O objetivo deste travalho foi avaliar os efeitos do núcleo homeopático no desenvolvimento de novilhos super jovens em sistema de confinamento utilizando dietas com diferentes inclusões de concentrado (48 e $100 \%$ de concentrado).

\section{MATERIALE MÉTODOS}

O trabalho foi realizado no Núcleo de Produção Animal (NUPRAN) da Universidade Estadual do Centro-Oeste (UNICENTRO), com objetivo de avaliar as variáveis correspondente ao desempenho de novilhos de corte terminados em confinamento. Os tratamentos constituíram-se 
de dieta $100 \%$ concentrado com ou sem homeopatia e dieta com 48 \% de concentrado mais silagem de milho com ou sem homeopatia.

O trabalho durou 119 dias, sendo 14 dias de adaptação e cinco períodos de 21 dias de avaliações. Utilizou-se 32 novilhos inteiros, da raça Charolês, provenientes do mesmo rebanho, com idade média de 12 meses e peso vivo inicial de $323 \pm 5 \mathrm{~kg}$ vermifugados e equilibrados por peso e condição corporal, onde a baia com dois animais representou uma unidade experimental.

Os animais foram pesados a cada 21 dias, após jejum de sólidos de 12 horas. Foi avaliado o consumo médio diário de matéria seca expresso em $\mathrm{kg}$.animal ${ }^{-1}$ (CMSD) e por $100 \mathrm{~kg}$ de peso vivo (CMSP), o ganho de peso médio diário (GMD) e conversão alimentar (CA). As avaliações das hipófises foram realizadas no laboratório de patologia clínica veterinária da UNICENTRO.

O manejo alimentar foi realizado duas vezes ao dia, às 6:00 e às 17:00 horas, sendo registrado diariamente o consumo voluntário da dieta. O produto homeopático utilizado foi o CONVERT $\mathrm{H}^{\circledR}$, sendo a dose recomendada de 100 g.animal.dia ${ }^{-1}$, praticando a adição á dieta do animal.

O delineamento experimental foi o inteiramente casualizado, composto por quatro tratamentos com quatro repetições, onde cada repetição constitui-se de uma baia com dois animais. Os dados coletados para cada variável foram submetidos a analise de variância com comparação de médias, a 5 \% de significância, por intermédio do programa estatístico SAS (1993). O experimento foi conduzido atendendo os princípios de bem estar e ética com animais experimentais sob aprovação da comissão de ética (CEUA/ UNICENTRO), conforme parecer do ofício 028/2011 de 15 de agosto de 2011.

\section{RESULTADOSEDISCUSSÃO}

Evidenciou-se (tabela I) um consumo de concentrado na ordem de 1,15\% e 1,83 \% do peso vivo dos animais, respectivamente para as dietas com $48 \%$ e $100 \%$ de concentrado. Houve interação significativa para GMD, CMSD e CMSP, enquanto que para CA não houve interação.

Animais alimentados com $48 \%$ de concentrado mais homeopatia tiveram maior $(\mathrm{p}<0,05)$ ganho de peso $\left(1,688 \mathrm{~kg} \cdot \mathrm{dia}^{-1}\right)$ comparativamente à dieta sem homeopatia $(1,379$ $\left.\mathrm{kg} \cdot \mathrm{dia}^{-1}\right)$, este não diferindo dos animais alimentados com $100 \%$ concentrado com $\left(1,424 \mathrm{~kg} \cdot \mathrm{dia}^{-1}\right)$ ou sem $\left(1,484 \mathrm{~kg} \cdot \mathrm{dia}^{-1}\right)$ homeopatia. Com relação ao CMSD e CMSP, maiores consumos foram observados na dieta com $48 \%$ de concentrado com homeopatia (9,86 kg.dia-1 e 2,29\% PV, respectivamente), assim como menores consumos foram observados na dieta com $100 \%$ concentrado com homeopatia (7,49 kg.dia ${ }^{-1}$ e $1,76 \% \mathrm{PV}$, respectivamente).

Houve diferença para CA $(\mathrm{p}<0,05)$ sob efeitos individuais para nível de concentrado e uso de homeopatia. Novilhos terminados em confinamento que receberam tratamento com homeopatia melhoraram a eficiência de transformação da matéria seca consumida em ganho de peso (5,90 contra 6,64) em relação aos não tratados, independente do nível de concentrado. De mesma forma, animais terminados em confinamento com dietas $100 \%$ concentrado também tiveram melhor CA $(p<0,05)$ comparativamente à dieta com $48 \%$ de concentrado (5,94 contra 6,59).

Segundo Katsuki (2009), o milho inteiro produz estímulo suficiente para ruminação e motilidade ruminal, permitindo eliminar a necessidade de fibra longa nas rações de alto teor de concentrado, no entanto, a inclusão de fontes de fibra não forragem pode melhorar o padrão da fermentação ruminal, manter o pH elevado e proporcionar maior consumo.

Ítavo et al. (2010), utilizando complexo homeopático promotor de crescimento na dose de 4 g.animal.dia-1 ${ }^{-1}$ evidenciaram maiores ganhos de peso em novilhos

Archivos de zootecnia vol. 63, núm. 241, p. 200. 
Brangus confinados suplementados com a homeopatia (1,350 vs 1,230 kg. animal.dia- $\left.{ }^{-1}\right)$ comparativamente ao tratamento controle, enquanto que para os parâmetros conversão alimentar $(8,19$ vs 8,66$)$ e consumo de matéria seca $\left(10,66\right.$ vs $\left.10,58 \mathrm{~kg} \cdot \mathrm{dia}^{-1}\right)$ o uso da homeopatia não apresentou resultados significativos, respectivamente. Segundo Paranhos da Costa e Costa-e-Silva (2007), a explicação para os resultados encontrados no presente trabalho está na possibilidade de se melhorar o desempenho dos animais quando empregadas alternativas de produtos que visam uma redução na reatividade do mesmo.

Tabela I. Desempenho de novilhos terminados em confinamento. (Performance of feedlot finished steers).

\begin{tabular}{|c|c|c|c|}
\hline & \multicolumn{2}{|c|}{ Homeopatia } & \multirow[b]{2}{*}{ Média } \\
\hline & Com & Sem & \\
\hline \multicolumn{4}{|l|}{ Peso vivo final, kg } \\
\hline $48 \%$ concentrado & $500^{a}$ & $468^{b}$ & 484 \\
\hline $100 \%$ concentrado & $473^{b}$ & $479^{b}$ & 476 \\
\hline Média & 486 & 473 & - \\
\hline \multicolumn{4}{|l|}{ GMD, kg.dia-1 } \\
\hline $48 \%$ concentrado & $1,688^{a}$ & $1,379^{b}$ & 1,534 \\
\hline $100 \%$ concentrado & $1,424^{b}$ & $1,483^{b}$ & 1,454 \\
\hline Média & 1,556 & 1,431 & - \\
\hline \multicolumn{4}{|l|}{ CMSD, kg.dia-1 } \\
\hline $48 \%$ concentrado & $9,66^{a}$ & $8,89^{b}$ & 9,47 \\
\hline $100 \%$ concentrado & $7,49^{d}$ & $7,77^{\mathrm{c}}$ & 7,67 \\
\hline Média & 8,72 & 8,43 & - \\
\hline \multicolumn{4}{|l|}{ CMSP, \% } \\
\hline $48 \%$ concentrado & $2,25^{a}$ & $2,09^{b}$ & 2,21 \\
\hline $100 \%$ concentrado & $1,76^{d}$ & $1,87^{\mathrm{c}}$ & 1,83 \\
\hline Média & 2,04 & 2,00 & - \\
\hline \multicolumn{4}{|l|}{ CA, CMSD/GMD } \\
\hline $48 \%$ concentrado & 5,95 & 6,95 & $6,59^{A}$ \\
\hline $100 \%$ concentrado & 5,64 & 6,17 & $5,94^{\mathrm{B}}$ \\
\hline Média & $5,90^{\mathrm{B}}$ & $6,64^{A}$ & - \\
\hline
\end{tabular}

CMS = Consumo de matéria seca; $\mathrm{GMD}=$ Ganho de peso médio diário; $\mathrm{CA}=$ Conversão alimentar.

$\mathrm{AB}$, abMédias com letras diferentes, na linha ou na coluna, diferem entre si (maiúsculas, pelo teste $\mathrm{F}$; minúsculas, Teste Tukey) $(p<0,05)$.
Os dados ainda mostram que em dietas contendo volumoso, a homeopatia mostrouse mais eficiente na evolução do ganho de peso diário dos novilhos confinados, enquanto que nas dietas $100 \%$ concentrado, mostrou-se eficiente na redução do consumo de MS diário, sem no entanto reduzir o ganho de peso diário dos animais.

Ueno (2012) avaliando dietas $100 \%$ concentrado e silagem de milho mais $5,5 \mathrm{~kg}$ de concentrado.animal.dia ${ }^{-1}$ na relação fixa de fornecimento na terminação de novilhos Canchim, não observou diferença entre os tratamentos para GMD (1,564 kg animaldia $^{-1}$ contra $1,461 \mathrm{~kg}$ animal.dia ${ }^{-1}$ ), porém, relatou que o fornecimento de dieta $100 \%$ concentrado aumentou em média 103 g.animal.dia ${ }^{-1}$, e que o ganho de peso de animais alimentados com $100 \%$ de concentrado foi mais estável conforme o avanço dos períodos de confinamento quando comparado aos animais que consumiram volumoso.

Katsuki (2009) avaliando níveis de inclusão de casca de soja em dieta isenta de volumoso em confinamento de bovinos Nelore de 24 meses de idade e peso vivo inicial de $460 \mathrm{~kg}$, sendo o nível zero composto com 85 \% de grãos de milho inteiros e 15 \% de núcleo protéico, encontrou valores de desempenho para o nível zero de $0,953 \mathrm{~kg}$ de GMD, 8,01 kg de CA, 6,99 kg de CMSD e 1,43 \% de CMSP. Tal discrepância nos resultados de desempenho do presente trabalho quando comparados os mencionados, pode estar relacionada a diferença de idade, peso inicial e grupamento genético dos animais.

Na tabela II pode ser evidenciado a média dos resultados obtidos na mensuração das hipófises dos bovinos. Pôde se evidenciar diferenças significativas entre os tratamentos com e sem homeopatia respectivamente, para comprimento $(19,45 \mathrm{~mm}$ vs 21,95 mm), largura máxima (14,70 mm vs $15,75 \mathrm{~mm})$ e peso $(3,199 \mathrm{~g}$ vs 3,367 g) de hipófise, não sendo encontrado diferenças estatísticas para a característica de altura até infundíbulo (13,30 mm vs 12,85 mm). 
Tabela II. Comprimento, largura máxima, altura até infundíbulo e peso de hipófises dos novilhos confinados. (Length, width, height and weight of pituitary gland of feedlot steers).

\begin{tabular}{|c|c|c|c|}
\hline & \multicolumn{2}{|c|}{ Homeopatia } & \multirow[b]{2}{*}{ Média } \\
\hline & Com & Sem & \\
\hline \multicolumn{4}{|l|}{ Comprimento, mm } \\
\hline $48 \%$ concentrado & 20,50 & 20,90 & $20,70^{A}$ \\
\hline $100 \%$ concentrado & 18,40 & 21,00 & $19,70^{A}$ \\
\hline Média & $19,45^{\mathrm{B}}$ & $21,95^{A}$ & \\
\hline \multicolumn{4}{|l|}{ Largura Máxima, mm } \\
\hline $48 \%$ concentrado & 14,70 & 15,40 & $15,05^{A}$ \\
\hline $100 \%$ concentrado & 14,70 & 16,10 & $15,40^{A}$ \\
\hline Média & $14,70^{\mathrm{B}}$ & $15,75^{\mathrm{A}}$ & - \\
\hline \multicolumn{4}{|c|}{ Altura até Infundíbulo, mm } \\
\hline $48 \%$ concentrado & 13,60 & 13,20 & $13,40^{A}$ \\
\hline $100 \%$ concentrado & 13,00 & 12,50 & $12,75^{A}$ \\
\hline Média & $13,30^{A}$ & $12,85^{A}$ & \\
\hline \multicolumn{4}{|l|}{ Peso, g } \\
\hline $48 \%$ concentrado & 3,436 & 3,332 & $3,384^{A}$ \\
\hline $100 \%$ concentrado & 2,962 & 3,402 & $3,182^{\mathrm{B}}$ \\
\hline Média & $3,199^{\mathrm{B}}$ & $3,367^{A}$ & \\
\hline
\end{tabular}

ABMédias, seguidas por letras maiúsculas diferentes, na linha ou na coluna, diferem entre si pelo teste $F(p<0,05)$.

Porém, quando comparadas às características da hipófise em relação a dieta, verificouse diferenças significativas apenas na questão de peso da glândula, sendo a dieta contendo $48 \%$ de concentrado apresentou

\section{BIBLIOGRAFIA}

Ítavo, L.C.V.; Dias, A.M.; Ítavo, C.C.B.F.; Ottoni, A.L.; Morais, M.G. e Torres Júnior, R.A.A. 2010. Homeopatia na terminação de novilhos em confinamento. Arch Zootec, 59: 225-232.

Katsuki, P.A. 2009. Avaliação nutricional, desempenho e qualidade da carne de bovinos alimentados com rações sem forragem, com diferentes níveis de substituição do milho inteiro por casca de soja. Tese (Doutorado em Ciência Animal). Universidade Estadual de Londrina. Londrina, PR. 55 pp.

Paranhos da Costa, M.J.R. e Costa-e-Silva, E.V. peso da hipófise 0,202 g mais pesada que a dieta com $100 \%$ de concentrado. Resultado este que possivelmente esteja ligado ao fato de os animais terminados com a dieta $48 \%$ de concentrado tenham apresentado um melhor desenvolvimento durante período de confinamento.

Assim, pode-se correlacionar a fisiologia da glândula com o produto homeopático utilizado no presente trabalho, há relatos (Real, 2008) de atuação no quesito reatividade animal, tornando-o menos reativo frente há situações estimuladoras de estresse, necessitando em menor quantidade, da atuação do eixo hipotalâmicohipofisário-adrenal, caracterizando hipertrofia da glândula em animais que não continham homeopatia em sua dieta.

\section{CONCLUSÕES}

Níveis de 48 \% de inclusão de concentrado na dieta de novilhos terminados em confinamento tiveram maiores ganhos de peso e melhor conversão alimentar com a inclusão da homeopatia. A homeopatia não manifestou resposta positiva em animais terminados em confinamento com dieta $100 \%$ concentrado. A dieta 100\% concentrado proporcionou um menor consumo de matéria seca dia, sendo refletido também em uma melhor conversão alimentar dos novilhos confinados.

2007. Aspectos básicos do comportamento social de bovinos. Rev Bras Reprod Anim, 31: 172-176.

Real, C.M. 2008. Homeopatia populacional - Fundamentos, ruptura de um paradigma. A Hora Veterinária, 28: 13-20.

Ueno, R.K. 2012. Avaliação bioeconômica da cultura do milho (Zea mays L.) utilizada sob diferentes formas na alimentação de novilhos em confinamento. Dissertação (Mestrado em Agronomia - Produção Vegetal). Universidade Estadual do Centro Oeste. Guarapuava-PR. 152 pp.

Archivos de zootecnia vol. 63, núm. 241, p. 202. 